\title{
Evaluation of STOX1 as a preeclampsia candidate gene in a population-wide sample
}

\author{
Katja Kivinen ${ }^{1}$, Hanna Peterson ${ }^{1}$, Leena Hiltunen ${ }^{5}$, Hannele Laivuori ${ }^{2,3}$, Sanna Heino ${ }^{4}$, \\ Inkeri Tiala ${ }^{2}$, Sakari Knuutila ${ }^{4}$, Vesa Rasi ${ }^{5}$ and Juha Kere*,1 \\ ${ }^{1}$ Department of Biosciences at Novum, Karolinska Institutet, Stockholm, Sweden; ${ }^{2}$ Department of Medical Genetics, \\ University of Helsinki, Helsinki, Finland; ${ }^{3}$ Department of Clinical Genetics, Helsinki University Central Hospital, \\ Helsinki, Finland; ${ }^{4}$ Laboratory of Cytomolecular Genetics, Department of Pathology, University of Helsinki, Helsinki, \\ Finland; ${ }^{5}$ Finnish Red Cross Blood Service, Helsinki, Finland
}

Preeclampsia is a common, pregnancy-specific vascular disorder characterised by hypertension and proteinuria. A recent report suggested association of the STOX1 gene on chromosome 10q22.1 with preeclampsia in the Dutch population. Here, we present a comprehensive assessment of STOX1 as a candidate gene for preeclampsia in the Finnish population by re-examining our previous genetic linkage analysis results for both chromosome 10 and paralogous loci, by genotyping representative markers in a nationwide data set, and by studying STOX 1 expression in placentas from preeclamptic and uncomplicated pregnancies. In conclusion, we are unable to validate STOX1 as a common preeclampsia susceptibility gene.

European Journal of Human Genetics (2007) 15, 494-497. doi:10.1038/sj.ejhg.5201788; published online 7 February 2007

Keywords: genetic association; gene expression; genetic linkage; pregnancy; complication

\section{Introduction}

Preeclampsia is a multifactorial, pregnancy-specific disorder characterised by hypertension and proteinuria. Several preeclampsia susceptibility genes have been suggested, but association studies have yielded conflicting results. A recent report suggested association of STOX1 gene on chromosome 10q22.1 with preeclampsia with seven coding variations co-segregating with the preeclamptic phenotype in the Dutch population. ${ }^{1}$ To test the general applicability of this finding, critical evaluation of the candidate gene is required in other populations. We have used three methods to assess STOX1 as a candidate gene for preeclampsia. Firstly, we tested haplotype-tagging markers of STOX1 in a well-powered nationwide cohort of Finnish

*Correspondence: Professor Juha Kere, Department of Biosciences at Novum, Karolinska Institutet, S-141 57 Huddinge, Sweden.

Tel: + 468608 9158; Fax: + 468774 5538;

E-mail: juha.kere@biosci.ki.se

Received 26 June 2006; revised 29 November 2006; accepted 10 January 2007; published online 7 February 2007 preeclampsia mothers and matched controls. Secondly, we studied the expression of STOX1 in placentas from preeclamptic and uncomplicated pregnancies. Thirdly, we re-evaluated our previous genetic linkage analysis results for both chromosome 10 and paralogous loci. Unfortunately, none of these tests showed any evidence in support of STOX1 as a preeclampsia susceptibility gene, and we are therefore unable to confirm the association of STOX1 with preeclampsia.

\footnotetext{
Materials and methods

Subjects

The nationwide cohort consisted of 340 preeclamptic cases and 357 controls recruited from 100000 consecutive pregnancies during 1997-1998. Controls were matched with cases for the province of residence, number of pregnancies, age of mother and number of foetuses. Phenotypes were ascertained by two clinicians, and further subdivided into preeclampsia $(n=245)$, eclampsia (five),
} 
superimposed preeclampsia (46) and pregnancy-induced hypertension (44). We used the definitions of the National High Blood Pressure Education Working Group on High Blood Pressure in Pregnancy. ${ }^{2}$

Placental samples were obtained immediately after delivery from eight primiparous preeclamptic women and six primiparous controls. The following exclusion criteria were applied in recruiting controls: diabetes, renal disease, twin pregnancy, smoking or any other condition known to affect the preeclampsia risk.

The study protocols were approved by the Ethics Committees of the Department of Obstetrics and Gynaecology at Helsinki University Central Hospital and Karolinska Institutet South. All participants gave their informed consent for the study.

\section{Genotyping}

Four variations within the coding region of STOX1 were mapped to reference SNPs in the NCBI dbSNP database (rs1341667, exon 2, Y153H; rs10509304, exon 3, L519L; rs10509305, exon 3, E608D; rs10509306, exon 3, N715N). The remaining three STOX1 coding variations appeared to be specific to the Dutch study and were named for this project as rsSTOX1_01 (exon 1, R18P), rsSTOX1_02 (exon 1, A43A) and rsSTOX1_03 (exon 3, N825I). An additional SNP (rs4472827) was selected for the $3^{\prime}$ end of the STOX1 gene based on its tagging properties in the CEPH reference material available via the HapMap project. $^{3}$

Genotyping was performed on the Sequenom platform as described previously, and genotypes were verified independently by two investigators. ${ }^{4}$ Hardy-Weinberg equilibrium was assessed for each marker as a quality control procedure. Of the seven variations, rsSTOX1_02 and rsSTOX1_03 failed assay quality controls before genotyping, and rsSTOX1_01 failed the genotyping with only one successful genotype call. These three markers were therefore excluded from the association study. As both markers for exon 1 were excluded from the analysis, we re-sequenced exon 1 in 17 preeclamptic women and 22 control women from the nationwide data set using previously described primers and running conditions. ${ }^{1}$

\section{Association analysis}

Haploview 3.2 software was used to assess linkage disequilibrium (LD) between markers and to calculate both single-marker and haplotype associations. ${ }^{5}$ Associations were tested by $\chi^{2}$ and corrected for overall significance using 10000 permutations.

Genetic Power Calculator (http://statgen.iop.kcl.ac.uk/ $\mathrm{gpc} /$ ) was used to evaluate our ability to detect association to the marker rs1341667. ${ }^{6}$ We used risk allele frequency of $32 \%$ as observed in our nationwide data set, and preeclampsia prevalence of $8 \%$ as recently reported in the Finnish population. $^{7}$
RNA isolation and expression analysis

After isolation (TRIzol kit; Invitrogen, Carlsbad, CA, USA) and purification (RNAeasy total RNA isolation kit; Qiagen, Hilden, Germany), $6 \mu$ g of total RNA was reverse transcribed, amplified, labelled according to the alternative protocol for one-cycle cDNA synthesis and hybridised on Affymetrix Human Genome U133 Plus 2.0 arrays (Affymetrix, Santa Clara, CA, USA). Arrays were stained and scanned according to the Affymetrix protocols.

Transcript levels were determined from data images with Affymetrix GeneChip ${ }^{\circledR}$ DNA analysis software, and further analysis of data was performed with the open-source Bioconductor software packages under the R environment for statistical computing. ${ }^{8}$

\section{Results}

Re-sequencing of STOX1 exon 1

We re-sequenced the first exon of STOX1 in 17 preeclamptic cases and 22 controls from the nationwide data set to ensure full coverage of the region that showed association in the Dutch study. The sequences were in full agreement with the reference human genomic sequence, and no variations were discovered in exon 1.

\section{Association analysis of STOX1 markers}

The five successful markers (rs1341667-rs4472827) had similar allele frequencies in Finland and in the CEPH reference material obtained from the HapMap project. They formed a single LD block with identical haplotypes in both data sets, and six common haplotypes with a frequency of at least 1\% (Figure 1). Results are shown in Table 1 for the preeclamptic case-control data set, but the analyses were also performed for the entire data set and subsets thereof by adding cases with PIH, superimposed preeclampsia or both conditions without any improvement in the results. In summary, none of the single markers or haplotypes tested showed significant association in the Finnish case-control data set.

We used Genetic Power Calculator ${ }^{6}$ to evaluate our ability to detect association to marker rs1341667, which had been proposed to contain a predominant mutation by the Dutch study. ${ }^{1}$ Specifically, as hypothesized by the Dutch group, a causal relationship of STOX1 required its presence as heterozygous in the preeclamptic mother, and we asked whether preeclamptic women from the population-wide sample carried any of the haplotypes more often than control women without preeclampsia. Under the parameters described in Materials and methods, we maintained $80 \%$ power to detect a relative risk of 1.6 at alpha $=0.05$.

\section{Expression analysis of STOX1 transcripts}

We examined STOX1 expression in RNA samples obtained from placentas from eight preeclamptic and six uncomplicated deliveries using Affymetrix Human 


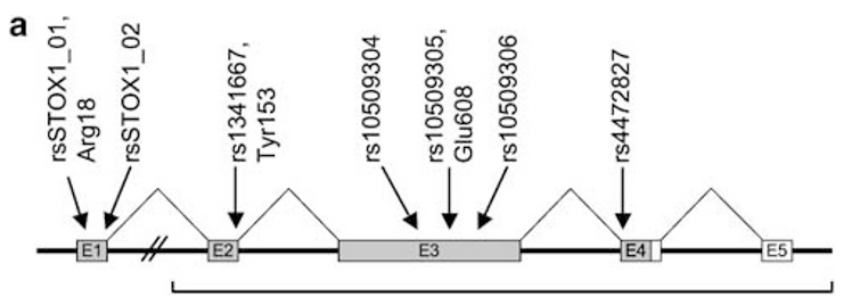

b

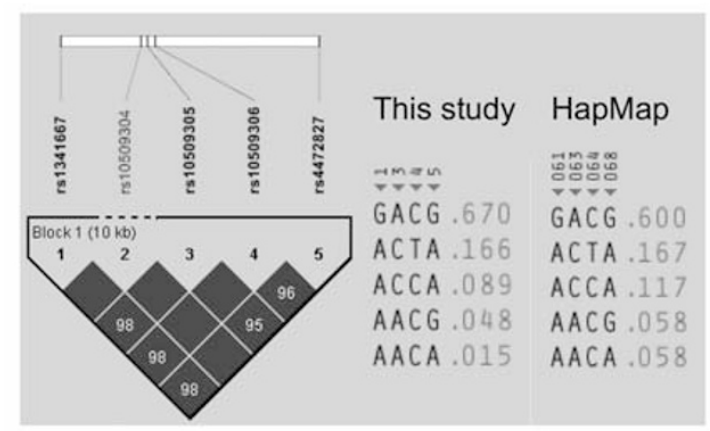

C

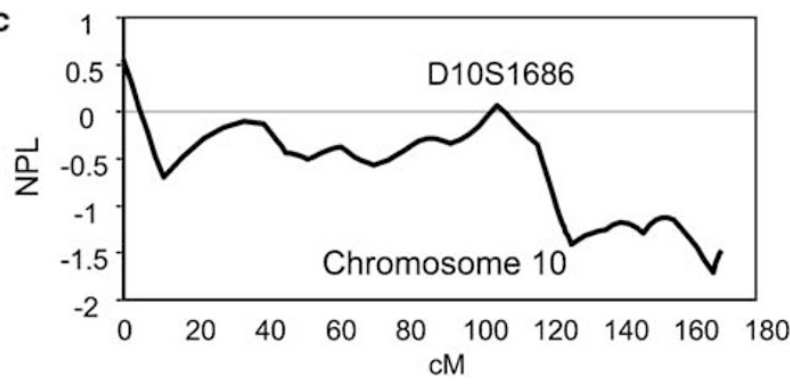

Figure 1 (a) Exonic architecture of the STOX1 gene representing the longest plausible transcript using evidence from public databases and a recent publication by van Dijk et al. ${ }^{1}$ The longest transcript spans $3.2 \mathrm{~kb}$ and contains 5 exons, but is missing $5^{\prime}$ UTR. The bracket indicates segment in high LD (haplotype block). (b) Representation of the LD structure in the STOX1 gene using $\mathrm{D}^{\prime}$ with $95 \% \mathrm{Cl}$ as described by Gabriel et al. ${ }^{11}$ Each square depicts the magnitude of LD for a pair of markers, red colour indicating high $\mathrm{D}^{\prime}$ and white colour low $\mathrm{D}^{\prime}$. (c). Plot of the linkage scores for chromosome 10 in the original 15 families from Laivuori et al. ${ }^{9}$ Marker D10S1686 showing the highest NPL score is $15 \mathrm{Mb}$ downstream of STOX1.
Genome-133Plus2.0. arrays. Neither of the two STOX1 specific probe sets present on this array showed statistically significant difference in expression levels between cases and controls, even when the two groups were subdivided according to delivery method (data not shown).

\section{Re-evaluation of Finnish linkage data}

We have previously published genome-wide linkage analysis of 15 Finnish multiplex families with severe preeclampsia. ${ }^{9}$ In the earlier report on STOX1, the cosegregation of the putative risk allele was observed in all affected sister pairs (eight informative sister pairs). ${ }^{1}$ Thus, this observation is compatible with genetic linkage in a mode considering sharing between affected individuals. A re-examination of linkage in our Finnish families with multiple affected women confirmed that in this data set, the linkage scores were negative or neutral (D10S1686, NPL-score 0.07 and $P$-value 0.75 ) throughout chromosome 10 (Figure 1).

It has been previously suggested that paralogous genes located on different chromosomes may be responsible for the same disease, although they are likely to segregate in different populations owing to different founders. ${ }^{10}$ Therefore, we searched for genes that had sequence similarity to STOX1 within the linkage peaks in our genome-wide scan. We did not find obvious paralogs to STOX1 within our linkage peak regions; the closest candidate was STOX2 (28\% identical to STOX1) on chromosome $4 \mathrm{q} 35$, which was located $>20 \mathrm{Mb}$ downstream from the $3^{\prime}$ end of our candidate region (using the LOD-1 cutoff to estimate borders for each linkage peak). If other paralogs exist, their sequences may have diverged too far from STOX1 to be recognised by standard similarity search methods.

\section{Discussion}

The suggestion of STOX1 as a susceptibility gene for preeclampsia requires follow-up studies in different popu-

Table 1 Genetic association analysis of STOX1 SNPs and haplotypes in Finnish preeclampsia mothers $(N=245)$ and matched controls $(N=357)$ recruited among 100000 consecutive pregnancies during 1997-1998

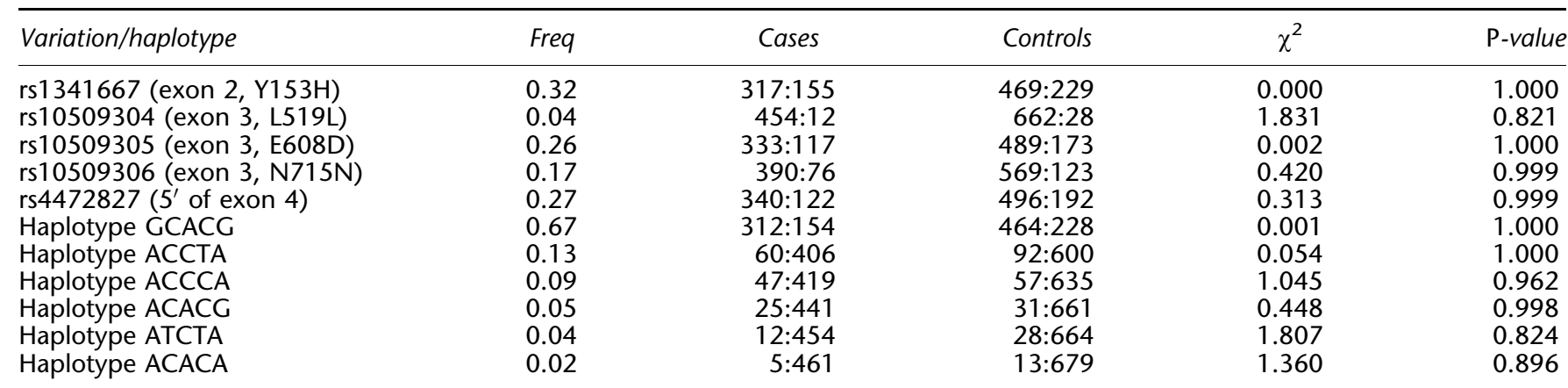

The association study has a power of $80 \%$ to detect a relative risk of 1.6 at alpha $=0.05$. Freq $=$ minor allele frequency for single markers and general frequency for haplotypes. 
lations and it is relevant to study its involvement using different methods. Therefore, we designed this study to test specifically several of the hypotheses put forward in the previous report. ${ }^{1}$ We tested genetic association in a population-wide sample set of preeclamptic women and carefully matched control women. Our power was sufficient to detect genetic effects with a relative risk of 1.6 or higher. Because a hypothesis of maternal effect and sharing between affected sisters was put forward, we evaluated our informative families for genetic linkage throughout chromosome 10, and also considered the existence of paralogous genes in the linkage peaks observed in our sample set. Finally, we compared the expression of STOX1 in freshly collected placental tissue samples from preeclamptic and uncomplicated pregnancies. None of these approaches revealed significant or even suggestive evidence in support of the involvement of STOX1 in preeclampsia.

It is evident that additional population samples are needed to increase the power further for detecting genetic effects that may be weaker than those that we could assess with good power. It is also possible that the specific mutations observed in the Dutch families ${ }^{1}$ are causal in only a subset of families, and therefore not easily detected by large population-based analyses. In that case, linkage approaches should be more powerful to implicate this locus, but at least in our family sample the linkage scores remained negative, yielding no support for the involvement of STOX1. Our analyses of STOX1 expression in placenta suggested that it is expressed at unchanged, stable levels in both preeclamptic and uncomplicated pregnancies.

In conclusion, STOX1 does not appear to represent a common preeclampsia susceptibility gene. To assess its role further, genetic tests in other population groups may be needed with subsequent meta-analysis to increase power for observing small genetic effects that our study may have missed.

\section{Acknowledgements}

We thank Hong Jiao, Leena Järvinen, Erja Kerkelä, Riitta Lehtinen, Cecilia M Lindgren and Ville Mäkelä for contributions to the laboratory analyses and all participants of the studies. This study was supported by Swedish Research Council, Academy of Finland, Sigrid Jusélius Foundation, Finnish Medical Foundation, Helsinki University Central Hospital Research Funds, Helsinki University Research Funds, Päivikki and Sakari Sohlberg Foundation and Uusimaa Regional Fund of the Finnish Cultural Foundation.

\section{References}

1 van Dijk M, Mulders J, Poutsma A et al: Maternal segregation of the Dutch preeclampsia locus at 10q22 with a new member of the winged helix gene family. Nat Genet 2005; 37: 514-519.

2 National High Blood Pressure Education Program Working Group: Report of the National High Blood Pressure Education Program Working Group on High Blood Pressure in Pregnancy. Am J Obstet Gynecol 2000; 183: S1-S22.

3 The International HapMap Consortium: The International HapMap Project. Nature 2003; 426: 789-796.

4 Ylisaukko-oja T, Peyrard-Janvid M, Lindgren CM et al: Familybased association study of DYX1C1 variants in autism. Eur J Hum Genet 2005; 13: 127-130.

5 Barrett JC, Fry B, Maller J, Daly MJ: Haploview: analysis and visualization of LD and haplotype maps. Bioinformatics 2005; 21: $263-265$.

6 Purcell S, Cherny SS, Sham PC: Genetic Power Calculator: design of linkage and association genetic mapping studies of complex traits. Bioinformatics 2003; 19: 149-150.

7 Kaaja R, Kinnunen T, Luoto R: Regional differences in the prevalence of pre-eclampsia in relation to the risk factors for coronary artery disease in women in Finland. Eur Heart J 2005; 26: $44-50$

8 Gentleman RC, Carey VJ, Bates DM et al: Bioconductor: open software development for computational biology and bioinformatics. Genome Biol 2004; 5: R80.1-R80.16.

9 Laivuori H, Lahermo P, Ollikainen V et al: Susceptibility loci for preeclampsia on chromosomes $2 \mathrm{p} 25$ and 9p13 in Finnish families. Am J Hum Genet 2003; 72: 168-177.

10 Leegwater PA, Vermeulen G, Konst AA et al: Subunits of the translation initiation factor eIF2B are mutant in leukoencephalopathy with vanishing white matter. Nat Genet 2001; 29: 383-388.

11 Gabriel SB, Schaffner SF, Nguyen $\mathrm{H}$ et al: The structure of haplotype blocks in the human genome. Science 2002; 296: $2225-2229$ 